\title{
Diagnose eines Adenokarzinoms
}

\author{
Ein Gynäkologe musste sich vor dem Landgericht Lüneburg \\ verantworten. Ihm wurde vorgeworfen, ein Adenokarzinom zu spät \\ erkannt zu haben.
}

m Sommer 2007 wurde die 1975 geborene Klägerin wegen therapieresistenter Zwischenblutungen vom sie betreuenden Frauenarzt zum beklagten Gynäkologen überwiesen. Wegen dieser Blutungsstörungen und dem Verdacht auf einen Endometriumpolypen führte der Gynäkologe im Herbst 2007 eine Hysteroskopie und eine fraktionierte Abrasio durch. Der histologische Befund ergab keinen Hinweis auf Malignität. Ein Ende 2007 genommener Abstrich war unauffällig. Im Frühjahr 2008 diagnostizierte der Gynäkologe eine blutungsbereite Ektopie und nahm wegen weiterer Blutungen eine Thermokoagulation vor. Im Sommer 2008 stellte sich die Patientin neuerlich mit Zwischenblutungen vor. Der Gynäkologe führte dies darauf zurück, dass die Patientin zuvor die Pille abgesetzt hatte. Im Herbst 2008 erfolgte nach weiteren Zwischenblutungen eine erneute Thermokoagulation. Ein Ende 2008 genommener Abstrich war unauffällig. Wegen weiter bestehenden Blutungsstörungen im Bereich des Muttermundes erfolgte im Frühjahr 2009 eine Loop-Exzision. Im pathologischen Befund wurde dargelegt, dass der Befund für einen HPV-assoziierten malignen Prozess, ausgehend von der Endozervix, spreche. Eine Zweitbefundung bestätigte ein hochdifferenziertes muzinöses endozervikales Karzinom. Im Anschluss erfolgte eine Hysterektomie nach Wertheim-Meigs. Die Histologie zeigte ein gut differenziertes Adenokarzinom der Zervix, auf diese beschränkt und vollständig im Gesunden entfernt.

Die Patientin warf dem Gynäkologen vor, er habe die Zwischenblutungen nicht als Hinweis auf ein Adenokarzinom bedacht und eine histologische $\mathrm{Ab}$ klärung zu spät veranlasst.

\section{So sah das Gericht den Fall}

Das Landgericht Lüneburg hat - sachverständig beraten - die Klage abgewiesen (Urt. v. 23.11.2011, Az. 2 O 274/10). Zwar sei davon auszugehen, dass das Karzinom schon 2007 bestanden habe und rückschauend betrachtet die Blutungen durch dieses Karzinom verursacht sein dürften. Aus der allein maßgeblichen Sicht ex ante hätte aber keine Indikation für weitere diagnostische Maßnahmen bestanden.

Die Früherkennung eines Adenokarzinoms sei im Vergleich zu einem Plattenepithelkarzinom im Bereich des Gebärmutterhalses erheblich erschwert. Zum einen seien drüsige Zellveränderungen durch Abstrichuntersuchungen oft nicht festzustellen, weil die Veränderungen in den Krypten abliefen und sich deshalb der oberflächigen Abstrichabnahme entzögen. Zum anderen käme es wesentlich seltener $\mathrm{zu}$ exophytischen Tumorbildungen, d.h. es gebe keine mit bloßem Auge erkennbaren Veränderungen, weil die Wucherungen oft nach innen wüchsen. Neben ungewöhnlichem Ausfluss, der bei der Patientin nicht vorgelegen habe, seien irreguläre Blutungen zwar ein klinisches Symptom für ein Zervixkarzinom. Gleichwohl hätten die Blutungen keine weitere Diagnostik indiziert. So sei nicht nur die Zytologie stets unauffällig gewesen, auch die histologische Untersuchung im Sommer 2007 habe keinen Hinweis auf Malignität ergeben. Hierauf habe sich der Gynäkologe verlassen dürfen und sei es nachvollziehbar, dass er von einer gutartigen Schleimhautveränderung ausgegangen war, die mit der Koagulation fachgerecht behandelt wurde.

Zwar hätte durch eine Konisation mit großer Wahrscheinlichkeit schon 2007

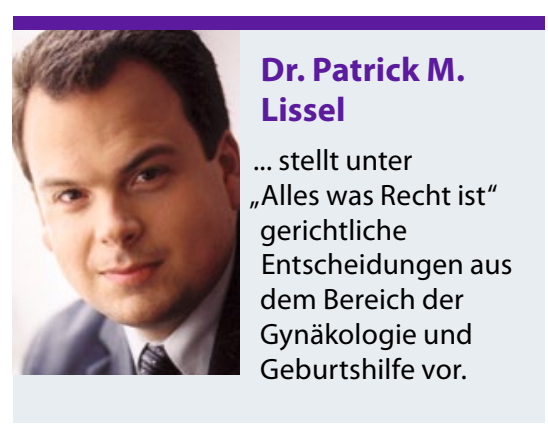

ein Adenokarzinom festgestellt werden können. Ohne objektive Anhaltspunkte, die den Verdacht einer bösartigen Veränderung begründen könnten, hätte es hierfür aber keine Indikation gegeben. Eine Konisation könne bei nachfolgenden Schwangerschaften zu erheblichen Problemen führen. Ohne einen entsprechenden Verdacht sei die Konisation trotz dauerhafter Blutungsstörungen als fehlerhaft anzusehen. Vor Sommer 2009 hätte auch kein weiterer histologischer Befund erhoben werden müssen. Ein Abstand von weniger als zwei Jahren sei keinesfalls als zu spät zu beurteilen.

\section{Was bedeutet das Urteil für den klinischen Alltag?}

Der Sachverhalt zeigt anschaulich, weshalb bei der Frage eines sorgfaltsgemäßen Verhaltens auf den Zeitpunkt der Behandlung abzustellen ist. Aus der Rückschau hätte das Karzinom durch andere Maßnahmen früher entdeckt werden können. Das Fehlen dieses nachträglichen Wissens kann man dem behandelnden Arzt nicht vorwerfen, solange es zum Zeitpunkt der Behandlung keine Anhaltspunkte gab, die zu einer weiteren Befunderhebung hätten führen müssen.

\section{Dr. Patrick M. Lissel, LL.M.}

Rechtsanwalt und Fachanwalt für

Medizinrecht

Ottostr. 1

80333 München 